\title{
CAN AlPhaFold2 PREDICT PROTEIN-PEPTIDE COMPLEX STRUCTURES ACCURATELY?
}

\author{
A PREPRINT \\ \begin{tabular}{|l|}
\hline \\
\hline Junsu Ko \\
\hline Arontier Co.
\end{tabular} \\ 15F, 241, Gangnam-daero, Seocho-gu, \\ Seoul, 15213, Republic of Korea \\ junsuko@arontier.co \\ ○ Juyong Lee \\ Department of Chemistry \\ Kangwon National University \\ Chuncheon, 24341, Republic of Korea \\ juyong. lee@kangwon.ac.kr
}

July 28, 2021

\begin{abstract}
In this preprint, we investigated whether AlphaFold2, AF2, can predict protein-peptide complex structures only with sequence information. We modeled the structures of 203 protein-peptide complexes from the PepBDB DB and 183 from the PepSet. The structures were modeling with concatenated sequences of receptors and peptides via poly-glycine linker. We found that for more than half of the test cases, AF2 predicted the bound structures of peptides with good accuracy, $\mathrm{C} \alpha$-RMSD of a peptide $<3.0 \AA$. For about $40 \%$ of cases, the peptide structures were modeled with an accuracy of $\mathrm{C} \alpha$-RMSD $<2.0 \AA$ A . Our benchmark results clearly show that AF2 has a great potential to be applied to various higher-order structure prediction tasks.
\end{abstract}

Keywords AlphaFold2, Protein-peptide complex structure modeling

\section{Introduction}

In CASP14, DeepMind introduced the version of their protein structure prediction model, AlphaFold2 (AF2), demonstrating that it can predict the protein structures with remarkable accuracy (Callaway [2020]). The median GDT-HA score of AF2 predictions was $78 \%$, which is comparable to crystal structures (Pereira et al.). This high accuracy indicates that almost all single-domain protein structures can be determined accurately with AF2. In addition, on July-23-2021, DeepMind and EMBL released the model structures of all human proteome and the other 20 organisms. These results will open new possibilities for biology and its related fields.

Based on the achievement of AF2, we tried to investigate whether AF2 can be applied beyond the prediction of single domain structures. As the first attempt, we evaluated AF2's ability to predict protein-peptide complex structures. Proteinpeptide complexes are playing essential roles in biological processes. Around $15 \tilde{4} 0 \%$ of protein-protein interactions (PPI) are estimated to be involved with protein-peptide interactions (Petsalaki and Russell [2008]). Therefore, the accurate modeling of protein-peptide complex structure is critical in understanding the mechanisms of biological reactions and development of peptide drugs (Ciemny et al. [2018]).

Various algorithms and programs have been developed to predict protein-peptide complex structures (Ciemny et al. [2018]). Protein-peptide modeling approaches can be categorized into three groups: (1) template-based (Lee et al. [2015]), (2) local docking (London et al. [2011]), and (3) global docking (Zhou et al. [2018]). A well-organized summary of protein-peptide modeling methods can be found in the reference (Ciemny et al. [2018]). Template-based methods generate model structures based on similar known complex structures. Thus, the approaches inherently rely on the existence of known structures and cannot be applied to novel targets. Local docking approaches perform peptide docking near a preset binding site provided by a user, which requires prior knowledge on a target protein.

Unlike template-based and local docking approaches, global docking approaches perform complex structure modeling solely based on a receptor structure and a peptide sequence. Thus, global docking approaches are general and can be 
bioRxiv preprint doi: https://doi.org/10.1101/2021.07.27.453972; this version posted July 28,2021 . The copyright holder for this preprint (which was not certified by peer review) is the author/funder, who has granted bioRxiv a license to display the preprint in perpetuity. It is made available under aCC-BY-NC-ND 4.0 International license.

applied to novel targets. However, due to a large degree of freedom, global docking approaches are computationally challenging and show relatively lower accuracy.

In this preprint, we investigated whether AF2 can predict protein-peptide complex structures only with sequence information. It has been shown that protein-protein complex structures can be modeled using coevolutionary information (Weigt et al. [2009], Schug et al. [2009], Balakrishnan et al. [2011]). Thus, if AF2 has learned how to extract coevolutionary information from multiple sequence alignment and interpret it to structural information succesfully, AF2 would be possible to predict the quaternary structures of proteins. Moriwaki and Ohue showed that AF2 may be able to predict the structures of protein-protein or protein-peptide complexes (Moriwaki, Ohue). In a similar vein, we assess the modeling power of AF2 for protein-peptide complexes by modeling the structures of 386 protein-peptide complexes after concatenating the sequences of receptors and peptides using a poly-glycine linker. Therefore, our benchmark corresponds to the global protein-peptide docking. We found that for more than half of the test cases, AF2 predicted the bound structures of peptides with good accuracy, C $\alpha$-RMSD of a peptide $<3.0 \AA$. Our test results clearly show that $\mathrm{AF} 2$ has a great potential to be applied to various higher-order structure prediction tasks.

\section{Methods and Materials}

\subsection{Dataset}

In this study, we used two sets of protein-peptide complexes. The first set consists of 203 protein-peptide complexes randomly selected from PepBDB (Wen et al. [2019]). We used the PepBDB release-2020-03-18 and applied the following two filtering criteria to select target complexes.

1. The sequence lengths of receptor and peptide should be greater than or equal to 10 .

2. Either of the sequence lengths of receptor and peptide should be greater than or equal to 50 .

3. There are no non-canonical amino acids.

The second set, PepSet, consists of 183 protein-peptide complexes, which were used for comprehensive benchmarking of protein-peptide docking programs (Weng et al. [2020]). The following filtering criteria were used to compile the set.

1. The peptide length ranges from 5 to 20 .

2. The resolution is $\leq 2.0 \AA$.

3. Peptides do not include non-canonical amino acids.

4. The sequence identity between any two protein monomers interacting directly with peptides is less than $30 \%$.

5. The bound structures have the corresponding unbound structures with a sequence identity $>90 \%$.

6. The RMSD of the backbone atoms of the residues in the bound structures within $10 A A$ from the peptide and the corresponding residues of the unbound structure is less than $2.0 \AA$.

\subsection{Modeling protein-peptide complex using AlphaFold2}

We performed protein-peptide complex modeling using AF2 (Jumper et al. [2021]) with a sequence that protein and peptide sequences were concatenated with 30 glycine residues. The source code of AF2 was retrieved from the github repository (https://github.com/deepmind/alphafold) on July-16-2021. No template information was used for structure modeling by setting the max_release_date parameter as 1979-Jan-01. The full_db preset was used for modeling, which is the eight times faster than the casp14 setting, which was used in CASP14. For all targets, five models were generated.

\subsection{Evaluation metrics}

We evaluated the quality of protein-peptide complex structures using the following three metrics: (1) complex RMSD, RMSD measured by the superposition of the whole protein-peptide complex (All_RMSD), (2) peptide RMSD, RMSD measured by the superposition of the peptide only (Pep_RMSD), and (3) peptide RMSD after the optimal superposition using a receptor structure (SP_RMSD, superposed peptide RMSD). In this study, we considered predictions with SP_RMSD $\leq 2.0 \AA$ are high-quality and with SP_RMSD $\leq 4.0 \AA$ near-native. SP_RMSD is conceptually similar to IL_RMSD used for the comprehensive benchmark (Weng et al. [2020]). All RMSD values were calculated with backbone C $\alpha$ atoms using the SVDSuperimposer implemented in BioPython (Cock et al. [2009]). No distance cutoff between a receptor and a peptide was considered for RMSD calculations, which may lead to high RMSD values for unsuccessful predictions. 


\section{Results and Discussion}

Table 1 shows the statistical summary of modeling quality of model 1 of the PepBDB set. It is noticeable that the median of SP_RMSD is $2.3 \AA$ indicating that more than half of predictions were near-native predictions. The first quartile values of SP_RMSD and All_RMSD are 0.79 and $1.22 \AA$ corresponding to highly accurate structures with atomic details. Among 203 targets, 90 complexes were predicted to be high quality, SP_RMSD $<2.0 \AA$ A. Also, in terms of All_RMSD, the median value is $2.61 \AA$ suggesting that the whole complex structure can be predicted with the current protocol accurately. In addition, the high accuracy of the models indicates that AlphaFold can be a tool for predicting peptide-binding regions without any prior information.

The results of the best model among five predictions in terms of All_RMSD are summarized on the right side of Table 1. When the best among five models are considered, the median of SP_RMSD and All_RMSD are 1.86 and $2.29 \AA$. With a threshold of SP_RMSD $<2.0 \AA, 104$ targets were predicted accurately among the 203 targets, corresponding to a success rate of $51 \%$.

Table 1: The summary of modeling quality of the PepBDB set

\begin{tabular}{c|ccc|ccc}
\hline & \multicolumn{3}{|c|}{ Top } & \multicolumn{3}{c}{ Best } \\
\hline & SP_RMSD & All_RMSD & Pep_RMSD & SP_RMSD & All_RMSD & Pep_RMSD \\
\hline Q1 & 0.79 & 1.22 & 0.99 & 0.75 & 1.20 & 1.07 \\
Q2 (median) & 2.30 & 2.61 & 2.09 & 1.86 & 2.29 & 1.91 \\
Q3 & 7.56 & 7.05 & 3.74 & 5.44 & 5.83 & 3.48 \\
min & 0.11 & 0.37 & 0.15 & 0.11 & 0.37 & 0.15 \\
max & 50.1 & 24.8 & 12.7 & 30.0 & 22.3 & 12.5 \\
\hline
\end{tabular}

The modeling results of PepSet are summarized in Table 2. Overall, RMSD values are similar to those of the PepBDB set. The median SP_RMSD of the top models is $2.88 \AA$, which is slightly worse than that of the PepBDB result. When the best models are considered, the SP_RMSD value decreases to $1.79 \AA$ A. In summary, it is clear that AF2 can predict protein-peptide complex structures accurately based on the modeling results of 386 protein-peptide complexes,

Table 2: The summary of modeling quality of the best model of PepSet

\begin{tabular}{c|ccc|ccc}
\hline & \multicolumn{3}{|c|}{ Top } & \multicolumn{3}{c}{ Best } \\
\hline & SP_RMSD & All_RMSD & Pep_RMSD & SP_RMSD & All_RMSD & Pep_RMSD \\
\hline Q1 & 0.82 & 1.52 & 0.91 & 0.66 & 1.39 & 0.87 \\
Q2 (median) & 2.88 & 3.63 & 1.92 & 1.79 & 2.45 & 1.94 \\
Q3 & 7.16 & 7.16 & 3.14 & 4.37 & 4.92 & 2.79 \\
min & 0.10 & 0.42 & 0.13 & 0.08 & 0.42 & 0.13 \\
max & 36.0 & 29.6 & 9.1 & 24.4 & 29.6 & 8.60 \\
\hline
\end{tabular}

Interestingly, modeling accuracy does not depend on the sequence length of a peptide or a complex. The SP_RMSD values and the peptide and complex sequence lengths of the PepBDB set models are illustrated in Figure 1 . It is observed that there is almost no correlation between the model accuracy and sequence lengths. This indicates that AF2 modeling does not rely on the number of contacts between a receptor and a peptide or shape complementarity, which is critical for many physics-based or empirical peptide docking programs. This result shows that the strength of the coevolutionary signal is independent of sequence lengths.

Two successful predictions are illustrated in Figure 2 In the case of 1B2D, the sequence lengths of receptor and peptide are similar. The modeled peptide structure is almost perfectly superposed with the crystal structure. In the case of 6RM8, a long peptide structure is similar to the crystal structure. The helical structure and the following disordered region of the peptide are closely following the crystal. Only the terminal region of the peptide deviates from the crystal.

Two unsuccessful prediction results are displayed in Figure 3 In the cases of 4C5A and 5GOW, AF2 could not find proper binding sites. The poly-glycine linker region is fully elongated, and a peptide is predicted to be far away from the receptor. AF2 may have predicted that the given receptor and peptide pairs would not bind. 
bioRxiv preprint doi: https://doi.org/10.1101/2021.07.27.453972; this version posted July 28, 2021. The copyright holder for this preprint (which was not certified by peer review) is the author/funder, who has granted bioRxiv a license to display the preprint in perpetuity. It is made available under aCC-BY-NC-ND 4.0 International license.

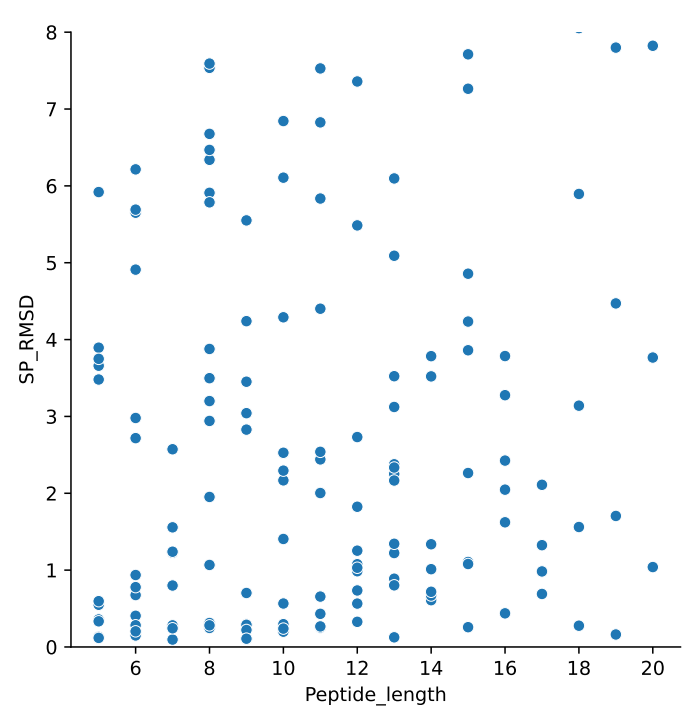

(a) Peptide length

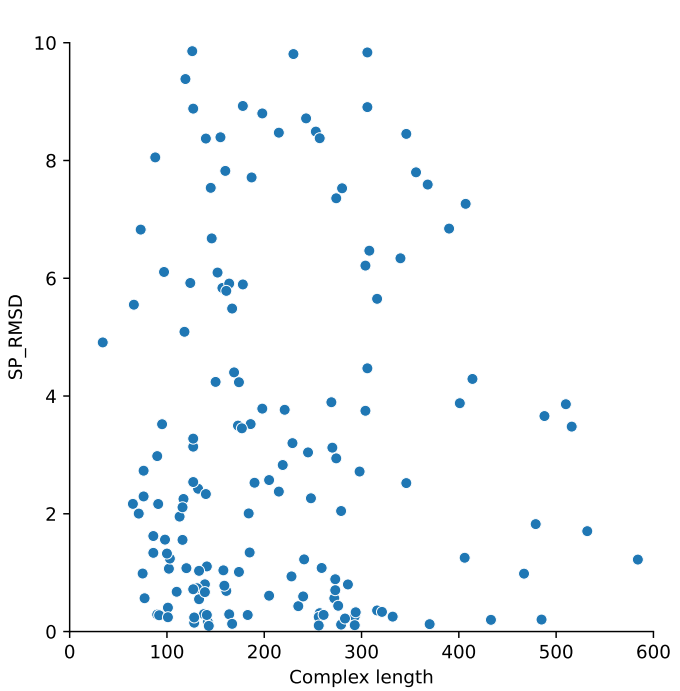

(b) Complex length

Figure 1: Relationship between model accuracy in SP_RMSD and (a) peptide and (b) complexe sequence lengths
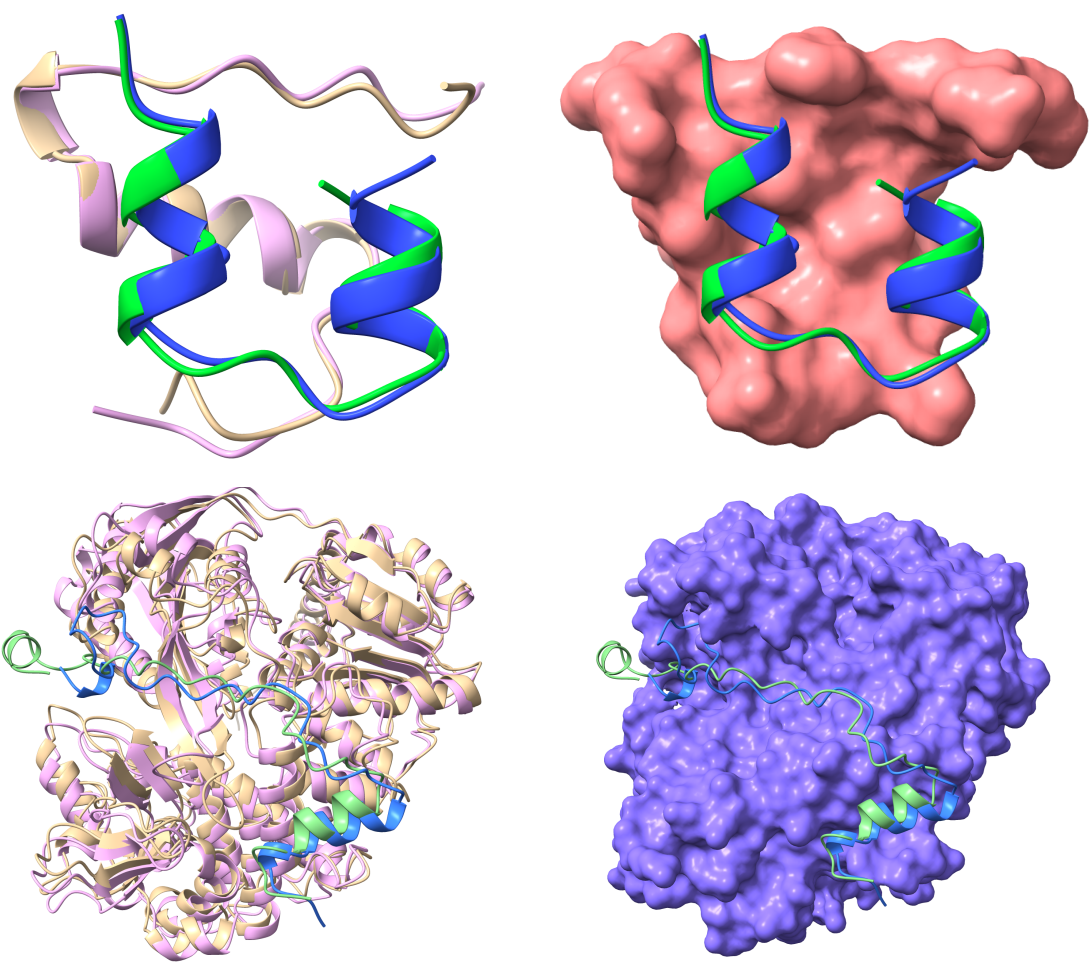

Figure 2: Modeling examples of successful predictions: (top) 1B2D and (bottom) 6RM8. The crystal structures of a receptor and a peptide are colored in gold and green. The model receptor and peptide structures are colored in pink and blue. The glycine linker is not displayed for clarity. 

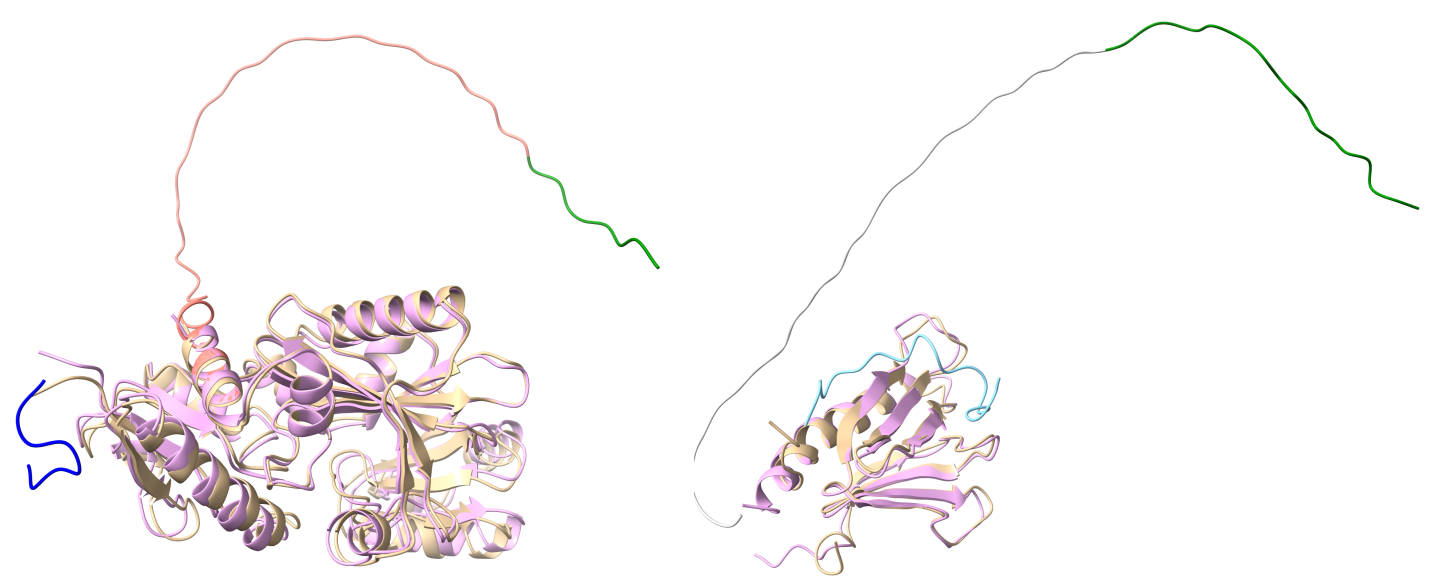

Figure 3: Modeling examples of bad predictions: (left) 4C5A and (right) 5GOW. The crystal structures of a receptor and a peptide are colored in gold and green. The model receptor and peptide structures are colored in pink and blue.

\section{Conclusion}

In this study, we showed that AF2 can predict the protein-peptide complex structures accurately without template information. It is remarkable that AF2 generated accurate model structures using only the concatenated sequence of a receptor and a peptide via a poly-glycine linker. When the top models are considered, $44 \%$ and $61 \%$ of the PepBDB set targets were predicted with accuracy of SP_RMSD $<2.0$ Anand SP_RMSD $<4.0 \AA$, respectively. This level of accuracy is comparable or better than existing protein-peptide modeling methods without using template information. However, we cannot completely rule out the possibility that AlphaFold may have used protein-peptide complex structures information during training. It is also worth investigating how much model qualities would improve with template information. These results suggest that AF2 learned how to extract interaction information between receptors and peptides from evolutionary information. In addition, our results indicate that AF2 is not just a modeling tool for monomeric structures, but also can be an extremely powerful tool for modeling more complex quaternary structures of proteins.

\section{References}

Ewen Callaway. 'it will change everything': Deepmind's ai makes gigantic leap in solving protein structures. Nature, pages 203-204, 2020.

Joana Pereira, Adam J. Simpkin, Marcus D. Hartmann, Daniel J. Rigden, Ronan M. Keegan, and Andrei N. Lupas. High-accuracy protein structure prediction in casp14. Proteins: Structure, Function, and Bioinformatics, n/a(n/a). doi:https://doi.org/10.1002/prot.26171. URL https://onlinelibrary.wiley.com/doi/abs/10.1002/prot. 26171.

Evangelia Petsalaki and Robert B Russell. Peptide-mediated interactions in biological systems: new discoveries and applications. Current Opinion in Biotechnology, 19(4):344-350, 2008. ISSN 0958-1669. doi:https://doi.org/10.1016/j.copbio.2008.06.004. Protein technologies / Systems biology.

Maciej Ciemny, Mateusz Kurcinski, Karol Kamel, Andrzej Kolinski, Nawsad Alam, Ora Schueler-Furman, and Sebastian Kmiecik. Protein-peptide docking: opportunities and challenges. Drug Discovery Today, 23(8):1530-1537, 2018. ISSN 1359-6446. doi https://doi.org/10.1016/j.drudis.2018.05.006. URL https://www . sciencedirect com/science/article/pii/S1359644617305937.

Hasup Lee, Lim Heo, Myeong Sup Lee, and Chaok Seok. GalaxyPepDock: a protein-peptide docking tool based on interaction similarity and energy optimization. Nucleic Acids Research, 43(W1):W431-W435, 2015. ISSN 0305-1048. doi:10.1093/nar/gkv495. URL https://doi.org/10.1093/nar/gkv495.

Nir London, Barak Raveh, Eyal Cohen, Guy Fathi, and Ora Schueler-Furman. Rosetta FlexPepDock web server-high resolution modeling of peptide-protein interactions. Nucleic Acids Research, 39(suppl 2$)$ : W249-W253, jul2011. ISSN0305 - 1048. doi : 10.1093/nar/gkr431. 
bioRxiv preprint doi: https://doi.org/10.1101/2021.07.27.453972; this version posted July $28,2021$. The copyright holder for this preprint (which

was not certified by peer review) is the author/funder, who has granted bioRxiv a license to display the preprint in perpetuity. It is made available under aCC-BY-NC-ND 4.0 International license.

arXiv Template

A PREPRINT

Pei Zhou, Botong Li, Yumeng Yan, Bowen Jin, Libang Wang, and Sheng-You Huang. Hierarchical Flexible Peptide Docking by Conformer Generation and Ensemble Docking of Peptides. Journal of Chemical Information and Modeling, 58(6):1292-1302, 2018. doi 10.1021/acs.jcim.8b00142. URL https://doi.org/10.1021/acs.jcim.8b00142

Martin Weigt, Robert A. White, Hendrik Szurmant, James A. Hoch, and Terence Hwa. Identification of direct residue contacts in protein-protein interaction by message passing. Proceedings of the National Academy of Sciences, 106(1): 67-72, 2009. ISSN 0027-8424. doi:10.1073/pnas.0805923106. URL https://www.pnas .org/content/106/1/67.

Alexander Schug, Martin Weigt, José N. Onuchic, Terence Hwa, and Hendrik Szurmant. High-resolution protein complexes from integrating genomic information with molecular simulation. Proceedings of the National Academy of Sciences, 106(52):22124-22129, 2009. ISSN 0027-8424. doi/10.1073/pnas.0912100106. URL https://www.pnas. org/content/106/52/22124.

Sivaraman Balakrishnan, Hetunandan Kamisetty, Jaime G Carbonell, Su-In Lee, and Christopher James Langmead. Learning generative models for protein fold families. Proteins: Structure, Function, and Bioinformatics, 79(4): 1061-1078, 2011.

Yoshitaka Moriwaki. https://twitter.com/Ag_smith/status/1417063635000598528?s=20. Accessed: 202107-28.

Masahito Ohue. https://twitter.com/tonets/status/1417363184927662080?s=20. Accessed: 2021-07-28.

Zeyu Wen, Jiahua He, Huanyu Tao, and Sheng-You Huang. Pepbdb: a comprehensive structural database of biological peptide-protein interactions. Bioinformatics, 35(1):175-177, 2019.

Gaoqi Weng, Junbo Gao, Zhe Wang, Ercheng Wang, Xueping Hu, Xiaojun Yao, Dongsheng Cao, and Tingjun Hou. Comprehensive evaluation of fourteen docking programs on protein-peptide complexes. Journal of chemical theory and computation, 16(6):3959-3969, 2020.

John Jumper, Richard Evans, Alexander Pritzel, Tim Green, Michael Figurnov, Olaf Ronneberger, Kathryn Tunyasuvunakool, Russ Bates, Augustin Žídek, Anna Potapenko, et al. Highly accurate protein structure prediction with alphafold. Nature, pages 1-11, 2021.

Peter JA Cock, Tiago Antao, Jeffrey T Chang, Brad A Chapman, Cymon J Cox, Andrew Dalke, Iddo Friedberg, Thomas Hamelryck, Frank Kauff, Bartek Wilczynski, et al. Biopython: freely available python tools for computational molecular biology and bioinformatics. Bioinformatics, 25(11):1422-1423, 2009. 